population with RA and a possible correlation with disease activity in a specialized rheumatology center.

Methods: A descriptive cross-sectional study was performed in a specialized clinic dedicated to care patients with rheumatoid arthritis (RA). Data was collected during a two year period at a psychology consultation, through semi-structured interview. Descriptive epidemiology was applied for continuous variables, using measures of central tendency and dispersion for categorical and qualitative variables by averages and percentages. We analyzed bivariate association with Pearson's $X^{2}$.

Results: We included 1398 patients attending to our psychology consultation. Mean age was 55 years $\pm 8 ; 80 \%$ were female and $20 \%$ male. Mean DAS28 was $2.6 \pm 1.3$, mean $\mathrm{HAQ}$ was $1.6 \pm 1.6$; patients had the disease for an average of 12 years $\pm 8 ; 41 \%$ of patients had comorbidities associated with non-autoimmune disease, $14 \%$ comorbidities related to autoimmune disease; $35 \%$ of our patients did not report other comorbidities. Most of patients were married $60 \%$, followed by divorced $19 \%$, single $14 \%$ and widowed $7 \%$. Regarding occupation $33 \%$ were employees, $25 \%$ were housekeepers or retired due to age, $12 \%$ were retired due to disabilities, and $3 \%$ unemployed. Of the total population $45 \%$ had elementary school, $32 \%$ high school, $8 \%$ college education, $7 \%$ graduate education and $7 \%$ were illiterate. $17 \%$ of patients lived alone. Concerning sexual disorders, $38 \%$ reported no to have any sexual activity, $32 \%$ reported to have a satisfactory sexual life, $11 \%$ dyspareunia, $9 \%$ had an unsatisfactory sexual life, $5 \%$ loss of desire, $3 \%$ premature ejaculation and $2 \%$ orgasmic decrease. Regarding predisposing factors of sexual disorders $63 \%$ of our population did not present any, $11 \%$ had insecurity related to the sexual role, $10 \%$ inadequate information relating sexuality, $10 \%$ infidelity and $6 \%$ physical and biological predisposing factors. Regarding precipitant factors of sexual disorders $63 \%$ report no to have any, $19 \%$ had biological or physical factors, $12 \%$ infidelity, $3 \%$ inadequate information and $3 \%$ insecurity related to de sexual role. There was no statistical association between disease activity and predisposing or precipitant factors.

Conclusions: We found that a third of patients with AR have sexual disorders and a high percentage reported not having any sexual activity. Also, it is important to have a multidisciplinary care team for the patient with RA, including a psychologist and a sexologist for managing this kind of illness in order to improve the life quality of patients.

Disclosure of Interest: None declared

DOI: 10.1136/annrheumdis-2017-eular.5635

\section{AB1236-HPR CONNECTION BETWEEN FUNCTIONAL ABILITY AND SOCIO-DEMOGRAPHIC DATA IN PATIENTS WITH RA IN SLOVENIA}

M. Pavic Nikolic, S. Kosir Jost. Department of Rheumatology, University Medical Centre Ljubljana, Ljubljana, Slovenia

Background: Rheumatoid arthritis (RA) is the most common chronic inflammatory arthritis, characterized by progressive, destructive course when left untreated, resulting in severe patient disability and significantly reduced quality of life. RA has an important impact on work ability and economic status of patients.

Objectives: In our study we were focused on specific consequences of arthritis in terms of functional ability and we were looking for correlation with sociodemographic data.

Methods: The study was conducted between January 2016 and May 2016 at the Department of Rheumatology, UMC Ljubljana and included patients with RA. Data were collected using Arthritis Impact Measurement Scales 2 (AIMS2) questionnaire.

Results: One hundred RA patients (76\% women, mean age (SD) of $61.1 \pm 15.29$ years) participated in the study. Disease duration was \pm 5.6 year. Most of our patients were married, retired with elementary education and lower incomes (Table1).

Table 1. Socio-demographic status of respondents

\begin{tabular}{lccccccr}
\hline \multicolumn{2}{c}{ Married status $\mathrm{n}$} & \multicolumn{2}{c}{ Education level/n} & \multicolumn{2}{c}{ Working status $/ \mathrm{n}$} & \multicolumn{2}{c}{ Monthly revenue/n } \\
\hline Married & 70 & Primary school & 40 & Employed & 27 & up to $500 €$ & 32 \\
Divorced & 3 & High school & 27 & Housekeeper & 2 & $500 €-900 €$ & 42 \\
Single & 6 & University & 27 & Student & 1 & $900 €-1300 €$ & 19 \\
Widowed & 19 & Master's degree & 3 & Unemployed & 5 & $1300 €-1700 €$ & 4 \\
Separate life & 2 & Doctor's degree & 3 & Retired & 65 & $1700 €$ and up & 3 \\
\hline
\end{tabular}

Respondents of our study described their functional ability good by the average of the entire sample 2.06. This value shows us a relatively good functional ability of patients most days or very often. Patients had some problems with function of hand and wrist in particular by opening jars (43\%) and on area walking and bending with intensive activity (42\%). Correlation between functional ability and socio-demographic data show us some statistical deviance in age group 41 to 50 year $(p=0.94)$, in patients with higher education level $(p=0.99)$ and in group of patients who monthly earns more then $900 €(p=0.96)$. But we didn't find any statistically significant difference in the entire sample including patients treated with biological drugs (functional ability patients on biologics $p=0.91$ ).

Conclusions: We found fairly good functional ability of patients; which might be explained by relatively short duration of RA. The most important functionalrelated issues from patient perspective were identified. Despite that we not find a statistically significant relationship between socio-demographic data and functional ability of patients, it is very important that we pay attention on socially unprivileged patients and we offer our support in managing disease.

Disclosure of Interest: None declared

DOI: 10.1136/annrheumdis-2017-eular.2523

\section{AB1237-HPR POSTURAL STABILITY AND ANKLE PROPRIOCEPTION IN DIFFERENT SUBGROUPS OF SUBJECTS WITH HALLUX VALGUS}

O. Aydoğdu, Z. Sarı, G.M. Polat. Physiotherapy and Rehabilitation, Marmara University, Health Sciences Faculty, Istanbul, Turkey

Background: Hallux valgus ( $\mathrm{HV}$ ) is lateral deviation of the great toe towards the second with subluxation of the first metatarsophalangeal joint and medial deviation of the first metatarsal (1). Increasing HV severity has been shown to negatively impact on health-related quality of life and self-reported function, and HV has been linked to increased falls risk in older adults (2). HV, in particular, is associated with poorer performance during postural stability and functional testing in older adults (3).

Objectives: Despite the findings of impaired postural stability in older adults with hallux valgus, the links between functional status and postural stability, ankle proprioception are not well established in HV. One of clinical significance of this study was to determine whether impaired postural stability was caused by deficits in ankle proprioception and impaired functional status in HV subjects. Our purpose of this study was to assess postural stability and ankle proprioception in different subgroups of HV. In this study, we hypothesized that subjects with severe deformity would exhibit poorer postural stability and ankle proprioception performance compared to subjects with mild and moderate deformity in HV.

Methods: Thirty-five adults diagnosed with unilateral HV according to the Manchester Oxford Foot Questionnaire deformation grade 2 and on were participated in the study. They were distributed among three groups: Mild (grade 2), moderate (grade 3), and severe deformity (grade 4). Functional status was measured with a disease specific score (the hallux valgus scale of the American Orthopaedic Foot and Ankle Society). While postural stability was measured with Pedalo Sensamove ${ }^{\circledR}$ System, ankle proprioception was measured with Biodex Balance System Pro 4.

Results: Subjects in HV with severe deformity group showed poorer postural stability performance than those in HV with mild $(p=0.024)$ and moderate $(p=0.039)$ deformity groups. However, there was no significant difference between the groups in ankle proprioception. There was a significant correlation between postural stability and functional status $(p<0.05, r=0.771)$ in all subjects with $\mathrm{HV}$. In addition, it was found that ankle proprioception was not correlated to functional status and postural stability $(p>0.05)$.

Conclusions: It has been concluded that HV with severe deformity affects postural stability according to the results of our study. On the other hand, ankle proprioception is not as an important predictor as postural stability for rehabilitation in different subgroups of HV. Therefore subjects in HV with severe deformity should be focused on stability exercises, in particular, in addition to home - based exercise programs, foot orthoses, footwear recommendations, and patient education in their rehabilitation.

References:

[1] Booth S, Bhosale A, Mustafa A, Shenoy R, Pillai A. Triple osteotomy for the correction of severe hallux valgus deformity: Patient reported outcomes and radiological evaluation. Foot (Edinb). 2016 Aug;28:30-35.

[2] Hurn SE, Vicenzino B, Smith MD. Functional impairments characterizing mild, moderate, and severe hallux valgus. Arthritis Care Res (Hoboken). 2015 Jan;67(1):80-8.

[3] Sadra S, Fleischer A, Klein E, Grewal GS, Knight J, Weil LS Sr, Weil L Jr, Najafi B. Hallux valgus surgery may produce early improvements in balance control: results of a cross-sectional pilot study. J Am Podiatr Med Assoc. 2013 Nov-Dec;103(6):489-97.

Disclosure of Interest: None declared

DOI: 10.1136/annrheumdis-2017-eular.4669

\section{AB1238-HPR PEOPLE'S PERCEPTIONS OF THEIR PHONE CALL WITH RHEUMA DIRECTLY, A RHEUMATIC DISEASES HELPLINE}

S. Arvidsson ${ }^{1,2}$, M. Nylander ${ }^{2}$, S. Bergman ${ }^{1,2,3} .{ }^{1}$ School of Health and Welfare, Halmstad University; ${ }^{2}$ Spenshult Research and Development Centre, Halmstad; ${ }^{3}$ The Sahlgrenska Academy, University of Gothenburg, Gothenburg, Sweden

Background: Information on rheumatic diseases is often complex to understand or scary, and additional support is often necessary. Rheuma Directly (RD) is a helpline with specially trained nurses on rheumatic diseases, funded by the Swedish Rheumatism Association and Spenshult Research and Development Centre. Little is known of how people calling a helpline perceive the contact.

Objectives: To describe the variation in how people perceive the contact with the helpline RD.

Methods: The study had a descriptive, qualitative design with a phenomenographic approach and was carried out by means of 27 semi-structured telephone interviews. The informants were 22 female and 5 men, and their ages ranged from 22 to 89 years (mean 54 years).

Results: The informants called RD when they had problems getting answers 